\title{
uiGarden, - An Online HCI Society in Two Languages
}

\author{
Christina Li \\ uiGarden.net \\ Christina.li@uigarden.net
}

\begin{abstract}
Garden, - a bilingual webzine, gives an opportunity between practitioners and researches working in user experience designer field from the Chinese and English speaking part of the world to exchange opinions and gain further knowledge within the field.
\end{abstract}

Keywords: Online HCI Society, bilingual webzine, China.

\section{Background}

China is predicted to become the biggest market leader and an economical giant in the foreseeable future. This is the reason why the world is focusing its attention and investments in China. A steady flow of Chinese nationals are still leaving China parallel to this. Domestic ventures in the West deal with customers who have Chinese backgrounds from China. It is due to these two factors that there is a major increase in demand for usability.

The experience of different cultures coming into contact with each other can be a positive influence in this modern life we are in. This involves intercultural exchanges, hence the term 'melting pot', as well as bewilderment, misunderstandings and miscommunication. Design professionals, whether from the West or from China, are encountering new challenges when creating usable and enjoyable user experience for their consumers. The demand of a place where user experience designers can easily exchange ideas and opinions is there. The benefit of gaining inside knowledge in the development and views of the other nation is huge.

uiGarden has started to meet this need. It was created from the teams' passion desire for user-centred design. These groups of practitioners and researchers, with professional technology and experience from the West and China, have committed themselves to act as a 'bridge' for the Western and Chinese HCI community. We understand the necessity for a free flow of exchange between the Western and Eastern usability practitioners. We plan for uiGarden to serve as an open platform, a possible opinion for fast and free information exchanges between professional people, who may not have met otherwise. uiGarden is unique in that it offers opportunity to discuss, explore and potentially resolve some of these differences. It can act as a catalyst to highlight the diversity of the websites between the West and the East.

\section{The Webzine}

The aim of the team is to offer fast and abundant exchange of information between the Western and Chinese design communities as well as to build a society that will 
eventually benefit both sides. The creation of uiGarden signifies a new phase in the way the West and East communicates.

For Western practitioners, the idea is to provide a space to communicate and exchange views between professionals. The website also serves as a portal in the user interface design industry in the Far East, allowing an insight into this increasingly vital market.

For Chinese practitioners, the site provides access to the latest developments in the West which includes Chinese translated articles from top Western experts and providing discussion boards for a closer look at each featured article.

The main contents are divided by categories including Opinion, Methods, Case studies and Reviews and Interviews with attention on: exploring theories and concepts that reflect current industrial practice, forecasts articles that refer to the challenges faced by our discipline, articles reflecting to the teaching of user-centred techniques and methodologies, case studies from projects showing the application of user-centred techniques from two communities, reviews of books, conferences, sites, software, tools and interactive projects, and interviews with leading experts in the field giving their point of view to professional issues.

Articles in this edition are in both Chinese and English. Discussion boards are available at the end of each article to allow readers to communicate with the author. Discussion threads are also translated into the other language enabling readers from the other community to also discuss the article with the author.

Apart from the direct discussion regarding the articles with their authors, we also offer forums in both languages for general discussions, focuses on a wide range of topics, therefore not limiting the discussion on just one article. Popular topics will also be translated in order for participants to know what the most discussed about issues are by their peers in the other community.

\section{The Outcome}

The aim of uiGarden is to facilitate information exchange and communication between the Western and Chinese user experience design communities. UiGarden was launched in January 2005 and it has achieved more than 550,000 page views since. In March 2007, the site has had more than 24,000 page views in total alone which averages over 500 unique visitors every day. This number is increasing at a fast rate with visitors coming from all over the world, of which $60 \%$ are from China and $40 \%$ from the rest of the world (data is for March 2007). uiGarden appears as a link to many well-known design and usability websites including HCI Bibliography, jnd.org, informationdesign.org, ixdg.org amongst many others. It has currently (April 2007) more than 2,000 subscribers by email, RSS and to its forums.

There is still much to learn from the project. It is our belief that Western designers will gain more ideas, when designing for global products, as more effective communication exists between the West and East. On the other side, learning usability theories and methods without the language barrier will also allow more people in China to understand and accept the concept, which will allow the Chinese usability and design industries to compete with the world. 